\title{
Global Stability Analysis on the Dynamics of an SIQ Model with Nonlinear Incidence Rate
}

\author{
Xiuxiang Yang ${ }^{1}$, Feng $\mathrm{Li}^{1}$, and Yuanji Cheng ${ }^{2}$ \\ ${ }^{1}$ Department of Mathematics, Weinan Normal University, Weinan 714000 Shaanxi, China \\ ${ }^{2}$ School of Technology Malmo University, SE-205 06 Malmo, Sweden \\ yangxiuxiang2000@yahoo.com.cn, lifeng5849@163.com, \\ yuanji.cheng@mah.se
}

\begin{abstract}
An SIQ epidemic model with isolation and nonlinear incidence rate is studied. We have obtained a threshold value $R$ and shown that there is only a disease free equilibrium point when $R<1$, and there is also an endemic equilibrium point if $R>1$. With the help of Liapunov function, we have shown that disease free- and endemic equilibrium point is globally stable.
\end{abstract}

Keywords: SIQ model, Isolation, nonlinear infectious rate, threshold, equilibrium point, global stability.

\section{Introduction}

In this paper, we shall as well exploit the Lyapunov direct method to study the global stability analysis on the dynamics of an SIQ model with nonlinear incidence rate. Assuming that the population has a very short immunity period, which is negligible, and whence there is a class of recovered and the models being studied here are of SIQ type. Assume that the total population is divided into: $S=S(t)$ susceptible, $I(t)$ infected, $\mathrm{Q}(\mathrm{t})$ isolated and $\mathrm{R}(\mathrm{t})$ recovered classes at time t. Let $\mathrm{A}$ be the constant migration rate of the population, and $\mathrm{d}$ be natural death rate of each kind, $\alpha_{1}$ and $\alpha_{2}$ be the infected death rate of infectious and isolated classes respectively, and $\delta$ be the transition rate from infected to isolated classes, $r, \varepsilon$ be immunity's lose rate of infected respectively isolated classes. We assume that the constants $d, \delta, A$ are positive, and $r, \varepsilon, \delta, \alpha_{1}, \alpha_{2}$ are nonnegative. Thus we consider the

$$
\left\{\begin{array}{c}
S^{\prime}(t)=A-\beta I S / 1+m I-d S+r I+\varepsilon Q \\
I^{\prime}(t)=\beta I S / 1+m I-\left(r+\delta+d+\alpha_{1}\right) I \\
Q^{\prime}(t)=\delta I-\left(\varepsilon+d+\alpha_{2}\right) Q
\end{array}\right.
$$


we see that $\mathrm{N}$ satisfies: $N^{\prime}(t)=A-d N-\alpha_{1} I-\alpha_{2} Q \leq A-d N$, thus

$$
N(t) \leq N_{0} e^{-d t}+\frac{A}{d}\left(1-e^{-d t}\right)
$$

It follows from the above estimates that the solutions of initial value problem for system

(1) exist on $[0,+\infty), D=\left\{(S, I, Q) \quad R_{+}^{3}: S, I, Q \quad 0, S+I+Q \square A / d\right\}$ is invariant subset of (1).

\section{Local Stability of Equilibrium}

To find the equilibrium of system (1), we get the equilibrium $P_{0}(A / d, 0,0)$ and $P_{1}\left(S_{1}, I_{1}, Q_{1}\right)$, let's define the threshold number for $R_{1}=\frac{d\left(r+\delta+d+\alpha_{1}\right)}{\beta A}$, Then we have that $P_{1}$ is in $R_{+}^{3}$ if and only if $R_{1}<1$. Here $S_{1}=\frac{\left(r+\delta+d+\alpha_{1}\right)}{\beta}\left(1+m I_{1}\right)$, $Q_{2}=\frac{\delta I_{1}}{\varepsilon+d+\alpha_{2}}, \quad I_{1}=\frac{\beta A-d\left(r+d+\delta+\alpha_{1}\right)}{m d\left(r+d+\delta+\alpha_{1}\right)+\beta\left(d+\alpha_{1}+\frac{\delta\left(d+\alpha_{2}\right)}{\varepsilon+d+\alpha_{2}}\right)}$

Theorem 1. For the system (1), the infection-free equilibrium point $P_{0}$ is locally asymptotically stable if $R_{1}>1$, If $R_{1}<1$, then the endemic equilibrium point $P_{1}$ is locally asymptotically stable and $P_{0}$ becomes unstable.

Proof. First we consider the system (1) and the infectious free equilibrium $P_{0}$, The Jacobian matrix $J\left(P_{0}\right)$, Which has eigenvalues:

$$
\lambda_{1}=-(d+\varepsilon)<0, \quad \lambda_{2}=\frac{\beta A}{d}-\left(r+\delta+d+\alpha_{1}\right), \quad \lambda_{3}=-\left(\varepsilon+d+\alpha_{2}\right)<0,
$$

When $R_{1}<1$, then $\lambda_{2}$ is also negative, and hence $P_{0}$ is locally stable.

In the $P_{1}, \beta S_{2}=\left(r+d+\delta+\alpha_{1}\right)\left(1+m I_{2}\right)$. Let $d_{i}=d+\alpha_{i}, i=1,2$

According to $J\left(P_{1}\right)$, the associated characteristic equation is $\lambda^{3}+b_{1} \lambda^{2}+b_{2} \lambda+b_{3}=0$, 


$$
\begin{aligned}
& b_{1}=\beta I_{2}+d\left(1+m I_{2}\right)+m I_{2}\left(r+\delta+d_{1}\right)+\left(\varepsilon+d_{2}\right)\left(1+m I_{2}\right)>0 \\
& b_{2}=\left(\beta I_{2}+d+d m I_{2}\right) m I_{2}\left(r+\delta+d_{1}\right)+\left(\beta I_{2}+d+d m I_{2}\right)\left(\varepsilon+d_{2}\right)\left(1+m I_{2}\right) \\
& +m I_{2}\left(r+\delta+d_{1}\right)\left(\varepsilon+d_{2}\right)\left(1+m I_{2}\right)+\beta I_{2}\left(\delta+d_{1}-r m I_{2}\right)>0 \\
& b_{3}=m I_{2}\left(\varepsilon+d_{2}\right)\left(1+m I_{2}\right)\left(r+\delta+d_{1}\right)\left(\beta I_{2}+d+d m I_{2}\right) \\
& +\beta I_{2}\left(\left(\varepsilon+d_{2}\right)\left(1+m I_{2}\right)\left(\delta+d_{1}-r m I_{2}\right)-\delta \varepsilon\left(1+m I_{2}\right)^{2}\right)>0
\end{aligned}
$$

To verify the Hurwitz condition $b_{1} b_{2}-b_{3}>0$, we first rewrite: $b_{1}=c_{10}+c_{11} m I_{2}$,

$$
b_{2}=c_{20}+c_{21} m I_{2}+c_{22}\left(m I_{2}\right)^{2}, b_{3}=c_{30}+c_{31} m I_{2}+c_{32}\left(m I_{2}\right)^{2}+c_{33}\left(m I_{2}\right)^{3} \text {, }
$$

Where $\quad c_{10}=d+\varepsilon+d_{2}+\beta I_{2} \quad, \quad c_{11}=d+r+\varepsilon+d_{2}+\delta+d_{1}$,

$$
\begin{aligned}
& c_{20}=d\left(\varepsilon+d_{2}\right)+\left(\varepsilon+d_{2}+\delta+d_{1}\right) \beta I_{2}, \\
& c_{21}=r d+\left(r+\delta+d_{1}\right)\left(\varepsilon+d_{2}\right)+\left(d+\beta I_{2}\right)\left(\varepsilon+d_{2}+\delta+d_{1}\right), \\
& c_{22}=\left(r+\delta+d_{1}\right)\left(\varepsilon+d_{2}\right)+d\left(r+\varepsilon+d_{2}+\delta+d_{1}\right), \\
& c_{30}=\left(\varepsilon d_{1}+\delta d_{2}+d_{1} d_{2}\right) \beta I_{2}, \\
& c_{31}=d\left(r+\delta+d_{1}\right)\left(\varepsilon+d_{2}\right)+2\left(\varepsilon d_{1}+\delta d_{2}+d_{1} d_{2}\right) \beta I_{2}, \\
& c_{32}=2 d\left(r+\delta+d_{1}\right)\left(\varepsilon+d_{2}\right)+\left(\varepsilon d_{1}+\delta d_{2}+d_{1} d_{2}\right) \beta I_{2}, \\
& c_{33}=d\left(r+\delta+d_{1}\right)\left(\varepsilon+d_{2}\right) .
\end{aligned}
$$

Then get

$$
\begin{aligned}
& b_{1} b_{2}-b_{3}=c_{10} c_{20}-c_{30}+\left(c_{10} c_{22}+c_{11} c_{20}-c_{31}\right) m I_{2}+ \\
& \left(c_{10} c_{22}+c_{11} c_{21}-c_{32}\right)\left(m I_{2}\right)^{2}+\left(c_{11} c_{22}-c_{33}\right)\left(m I_{2}\right)^{3} \\
& C_{0}=: c_{10} c_{20}-c_{30}>0, C_{1}=: c_{10} c_{22}+c_{11} c_{20}-c_{31}>0 \\
& C_{2}=: c_{10} c_{22}+c_{11} c_{21}-c_{32}>0, \quad C_{3}=: c_{11} c_{22}-c_{33}>0, \text { So } P_{1} \text { is locally }
\end{aligned}
$$
asymptotically stable.

\section{Global Stability of Equilibrium}

Theorem 2. For the system (1), If $R_{1}>1$, then the disease free equilibrium $P_{0}$ of (2) is globally asymptotically stable; when $R_{1}<1$, then $P_{0}$ is unstable, and the endemic equilibrium point $P_{1}$ is globally asymptotically stable. 
Proof. we can easily proof the disease free equilibrium $P_{0}$ of (1) is globally asymptotically stable same as theorem. Following discuss asymptotic stability of $P_{1}$, we again change variable $N=S+I+Q$

$$
\begin{aligned}
& \left\{\begin{array}{c}
N^{\prime}(t)=A-d N-\alpha_{1} I-\alpha_{2} Q \\
I^{\prime}(t)=\frac{I}{1+m I}\left[\beta(N-I-Q)-\left(r+\delta+d+\alpha_{1}\right)(1+m I)\right] \\
Q^{\prime}(t)=\delta I-\left(\varepsilon+d+\alpha_{2}\right) Q
\end{array}\right. \\
& x=N-N_{1}, y=I-I_{1}, z=Q-Q_{1} \text {, then we deduce } \\
& \left\{\begin{array}{c}
x^{\prime}(t)=-d x-\alpha_{1} y-\alpha_{2} z \\
y^{\prime}(t)=\frac{y+I_{2}}{1+m\left(y+I_{2}\right)}\left(\beta x-\left(\beta+m\left(r+d+\delta+\alpha_{2}\right)\right) y-\beta z\right) \\
z^{\prime}(t)=\delta y-\left(\varepsilon+d+\alpha_{2}\right) z
\end{array}\right.
\end{aligned}
$$

We have used the relation $\left(r+\delta+d+\alpha_{1}\right)\left(1+m I_{2}\right)=\beta S_{2}=\beta\left(N_{2}-I_{2}-Q_{2}\right)$ Choose

the

Liapunov

function

$$
\begin{gathered}
V=\frac{\omega_{1} x^{2}}{2}+\omega_{2}\left(y-I_{2} \ln \left(1+\frac{y}{I_{2}}\right)+\frac{m y^{2}}{2}\right)+\frac{\omega_{3} z^{2}}{2}+\frac{(x-z)^{2}}{2} \\
\left.\frac{d V}{d t}\right|_{(3)}=\omega_{1} x\left(-d x-\alpha_{1} y-\alpha_{2} z\right)+\omega_{2} y\left(\beta x-\left(\beta+m\left(r+d+\delta+\alpha_{2}\right)\right) y-\beta z\right) \\
\quad+\omega_{3} z\left(\delta y-\left(\varepsilon+d+\alpha_{2}\right) z\right)+(x-z)\left(-d x-\left(\delta+\alpha_{1}\right) y+(\varepsilon+d) z\right) \\
=-d\left(1+\omega_{1}\right) x^{2}+\left(\omega_{2} \beta-\omega_{1} \alpha_{1}-\alpha_{1}-\delta\right) x y+\left(\varepsilon+2 d-\alpha_{2} \omega_{1}\right) x z-\omega_{2}\left(\beta+m\left(r+d+\delta+\alpha_{2}\right)\right) y^{2} \\
\quad+\left(\alpha_{1}+\delta+\omega_{3} \delta-\omega_{2} \beta\right) y z-\left(\varepsilon+d+\left(\varepsilon+d+\alpha_{2}\right) \omega_{3}\right) z^{2}
\end{gathered}
$$

If we choose $\omega_{1}, \omega_{2}, \omega_{3}>0 \quad \omega_{1}=(\varepsilon+2 d) / \alpha_{2}, \omega_{2}=\left(\omega_{1} \alpha_{1}+\alpha_{1}+\delta\right) / \beta, \omega_{3}=\omega_{1} \alpha_{1} / \delta$, $\left.\frac{d V}{d t}\right|_{(3)}=-d\left(1+\omega_{1}\right) x^{2}-\omega_{2}\left(\beta+m\left(r+d+\delta+\alpha_{2}\right)\right) y^{2}-\left(\varepsilon+d+\left(\varepsilon+d+\alpha_{2}\right) \omega_{3}\right) z^{2}$

Which is clearly negative definite. Hence, $P_{1}\left(S_{1}, I_{1}, Q_{1}\right)$ is globally asymptotically stable. 


\section{References}

1. Hethcote, H.W., van den Driessche: Some epidemiological models with nonlinear incidence rate. J. Math. Biol. 29, 271-287 (1991)

2. Li, M.Y., Muldowney, J.S.: Global stability of the SEIR model in epidemiology. Math. Biosci. 125, 155-164 (1995)

3. Ruan, S., Wang, W.: Dynamical behavior of an epidemic model with a nonlinear incidence rate. J. Diff. Equa. 188, 135-163 (2003)

4. Korobeinikov, A., Maini, P.K.: Nonlinear incidence and stability of infectious disease models. Math. Medicine and Biology 22, 113-128 (2005)

5. Kyrychko, Y.N., Blyuss, K.B.: Global properties of a delayed SIR model with temporary immunity and nonlinear incidence rate. Nonlinear Analysis: Real world Applications 6, 495-507 (2005)

6. Feng, Z.L., Thieme, H.R.: Recurrent outbreaks of childhood diseases revisited: The impace of isolation. Math. Biosci. 128, 93-130 (1995)

7. Gerberry, D.J., Milner, F.A.: An SEIQR model for childhood diseases. Mathematical Biology 59, 535-561 (2009)

8. Castillo-Chavez, C., Castillo-Garsow, C.W., Yakubu, A.A.: Matheamtical models of isolation and quarantine. JAMA 290, 2876-2877 (2003)

9. Xiao, D., Ruan, S.: Global analysis of an epidemic model with nonmonotone incidence rate. Math. Biosci. 208, 419-429 (2007)

10. Chinviriyasit, S., Chinviriyasit, W.: Global stability of an SIQ epidemic model. Kasetsart J., (Nat. Sci.) 41, 225-228 (2007) 